\title{
A Dissociation of Encoding and Retrieval Processes in the Human Hippocampus
}

\author{
Laura L. Eldridge, ${ }^{1}$ Stephen A. Engel, ${ }^{1}$ Michael M. Zeineh, ${ }^{2}$ Susan Y. Bookheimer, ${ }^{2}$ and Barbara J. Knowlton ${ }^{1}$ \\ ${ }^{1}$ Department of Psychology and 2Division of Brain Mapping, University of California, Los Angeles, Los Angeles, California 90095
}

The hippocampal formation performs two related but distinct memory functions: encoding of novel information and retrieval of episodes. Little evidence, however, resolves how these two processes are implemented within the same anatomical structure. Here we use high-resolution functional magnetic resonance imaging to show that distinct subregions of the hippocampus are differentially involved in encoding and retrieval. We found that regions early in the hippocampal circuit (dentate gyrus and CA fields 2 and 3 ) were selectively active during episodic memory formation, whereas a region later in the circuit (the subiculum) was active during the recollection of the learning episode. Different components of the hippocampal circuit likely contribute to different degrees to the two basic memory functions.

Key words: learning; memory; fMRI; CA3; subiculum; recollection

\section{Introduction}

Patients with hippocampal lesions are impaired at creating new memory representations of information in the environment (Zola-Morgan et al., 1986; Rempel-Clower et al., 1996). However, damage to the hippocampus not only causes deficits in learning but also in retrieval, particularly for memories from the recent past (Reed and Squire, 1998; Kopelman et al., 1999) and memories with episodic content (Vargha-Khadem et al., 1997; Gadian et al., 2000). Consistent with both of these functions, neuroimaging studies have shown that, in some cases, hippocampal activity reflects encoding processes (Stern et al., 1996; Brewer et al., 1998; Wagner et al., 1998), whereas in other cases it appears to reflect recollective processes (Henson et al., 1999; Eldridge et al., 2000).

It seems likely that these different processes are subserved by the individual subregions of the hippocampus, but functional evidence for regional specialization remains sparse. According to one view, the encoding and retrieval of memories are supported by different loci along the anteroposterior axis of the hippocampus (Lepage et al., 1998; Schacter and Wagner, 1999), although more recent studies failed to find these differences (Schacter et al., 1999; Greicius et al., 2003). In the present study, we adopt the new approach of examining the role of different hippocampal cell fields in encoding and retrieval. The hippocampus is primarily organized as a unidirectional circuit: information from entorhi-

Received Nov. 19, 2003; revised Jan. 27, 2005; accepted Jan. 28, 2005.

This work was supported by National Institutes of Health-National Institute on Aging Grant 441587-HB-33589. We thank John Mazziotta, the University of California, Los Angeles Brain Mapping Medical Organization, the Ahmanson Foundation, the Pierson-Lovelace Foundation, the Tamkin Foundation, and the Jennifer Jones-Simon Foundation for their support. We also thank Mark Cohen for assistance with fMRI and Russell Poldrack and Dean Buonamano for helpful discussions.

Correspondence should be addressed to Barbara J. Knowlton, Department of Psychology, University of California, Los Angeles, Franz Hall, Los Angeles, CA 90095. E-mail: knowlton@psych.ucla.edu.

DOI:10.1523/JNEUROSCI.3420-04.2005

Copyright $\odot 2005$ Society for Neuroscience $\quad$ 0270-6474/05/253280-07\$15.00/0 nal cortex converges on the dentate gyrus, which in turn projects to field CA3, which sends projections to field CA1. The circuit is completed as CA1 projects to the subiculum, the major output region of the hippocampus. These subregions differ in their cellular organization and connectivity (Amaral, 1993) and, thus, potentially make distinct contributions to the known stages of memory processing.

The present experiment tests for functional differences between hippocampal regions during episodic encoding and retrieval. We used high-resolution event-related functional magnetic resonance imaging (fMRI) to measure activity in distinct hippocampal subregions during learning and later retrieval of memories. This approach provided the anatomical precision needed to observe activation at different points in the hippocampal circuit as memories were formed and subsequently recollected. During encoding sessions, subjects studied word and picture pairs. During retrieval sessions, subjects judged whether presented words were previously studied or not and then classified their memory experience (using the remembered-knew task; see Materials and Methods). Data from both sessions were separately averaged within medial temporal lobe (MTL) regions, according to whether the subject reported episodic recollection, familiarity-based recognition, or the failure to retrieve a memory.

\section{Materials and Methods}

Subjects. Ten volunteers (age range, 21-29 years) with no history of neurological disorders were run in the experiment under a protocol approved by the University of California, Los Angeles Office for the Protection of Research Subjects. All subjects were right-handed, with an Edinburgh Handedness score of at least 16 of 20 . Subjects were paid $\$ 60.00$ for participation. Because of excessive head motion, one subject's data from encoding and one subject's data from retrieval were discarded. The data from these two subjects were included in the analyses of the other phase of the experiment.

Encoding. The encoding stimuli consisted of 99 unrelated pictures of 
object pairs with the corresponding object names printed below them on the screen (see Fig. 1). There were 11 trials in each of the nine encoding scans. Each trial consisted of $5 \mathrm{~s}$ of stimulus presentation, followed by $12.5 \mathrm{~s}$ of fixation. The pictures were simple line drawings (Snodgrass and Vanderwart, 1980), and the pairs were oriented either horizontally or vertically on the screen (the orientation varied randomly by trial). The first word of the pair was defined as the cue word, and the corresponding cue object appeared at the top or left position relative to the other object. The cue object was presented in one of four colors: blue, orange, pink, or green. Each color was presented an approximately equal number of times throughout the encoding phase. The noncue objects were presented in black and white. During the encoding phase, subjects were instructed to try to remember as many details from each trial as possible. Subjects were also informed that their memories for the stimuli would be tested later. No overt responses were made by the subject during the encoding phase of scanning.

Retrieval. Retrieval scans occurred $24 \mathrm{~h}$ after the encoding scanning session. The retrieval stimuli consisted of the 99 cue words and 45 lure (unstudied) words. In each of nine functional scans, 11 cue words and five lure words were presented in a random order. Target and lure items were counterbalanced across subjects. Before scanning, subjects were instructed that they would see a series of words and that, for each word, they would decide whether they confidently recognized the word as having been presented during the encoding session. For each recognized item, they would indicate whether they "remembered" the moment they had encountered the item during the first session (R) or if they simply "knew" the item was old because of a feeling of familiarity (K). The instructions were similar to the two-step instructions used by Eldridge et al. (2002). In each $5 \mathrm{~s}$ trial during scanning, the subject was first prompted to decide whether or not they recognized the item $(3 \mathrm{~s})$ and whether they remembered or knew it ( $2 \mathrm{~s})$. Responses were recorded via button presses. If the item was not recognized, the subject was allowed to press either button at the second prompt. Between trials, subjects maintained fixation for $12.5 \mathrm{~s}$. Subjects were instructed to disengage from retrieval performance during the fixation period.

After the retrieval scanning session, a posttest was conducted outside of the scanner to probe which stimulus details the subject was able to retrieve. For each studied cue word, subjects chose which of the two possible picture orientations (above or beside) and four possible picture colors (blue, orange, pink, or green) had been present during study. Subjects also attempted to recall the word that had been paired with the test word.

fMRI methods. A 3 T GE Signa scanner with ANMR (Wilmington, MA) echo-planar upgrade was used for all functional imaging. A bite bar minimized head motion during scanning. A sagittal localizer scan was used to identify the long axis of the hippocampus. High-resolution T2 fast spin echo images were acquired in an oblique coronal plane perpendicular to the long axis of the hippocampus for use in the segmentation process [repetition time (TR), $3000 \mathrm{~ms}$; echo time (TE), $41 \mathrm{~ms}$; field of view (FOV), 20 ; $512 \times 512$; echo train length, 8 ; number of excitations (NEX), 2; 16 slices, $3 \mathrm{~mm}$ thick, $0 \mathrm{~mm}$ space; voxel size, $0.391 \times 0.391 \times$ $3.00 \mathrm{~mm}$ ]. These 16 slices spanned the head, body, and most of the tail of the hippocampus. A T2*-weighted gradient echo sequence was used to measure blood-oxygen level-dependent (BOLD) contrast (TR, $3500 \mathrm{~ms}$; TE, $30 \mathrm{~ms}$; FOV, 20; $128 \times 128$; 11 slices, $3 \mathrm{~mm}$ thick, $0 \mathrm{~mm}$ space).

Motion correction was performed by aligning all functional volumes to the first volume time point using AIR software (Woods et al., 1999). To correct for distortions in the functional images attributable to echoplanar imaging (EPI), we followed the procedures outlined by Zeineh et al. (2000). Specifically, we acquired EPI anatomies (spin echo; TR, 4000 ms; TE, 54 ms; FOV, 20; $128 \times 128$; NEX, 4; 16 slices, 3 mm thick, $0 \mathrm{~mm}$ space) coplanar to our functional data that had distortions that closely matched those in the functional data. We next outlined the cortical manifold by hand within the EPI anatomies and shifted the EPI anatomies so that the manifold overlaid the cortical sheet visible in the non-EPI anatomies used for unfolding. Finally, we applied this shift to the functional data to register them with the non-EPI anatomies.

Flattened maps of the MTL were created for each hemisphere in each participant to trace regions of interest (ROIs) smoothly across scan planes. The ROIs contained anatomical subregions of the MTL and were drawn by hand using landmarks that identify the boundaries between regions (Zeineh et al., 2000, 2001). No such landmarks exist to segregate the CA2 and CA3 fields and the dentate gyrus (DG), and so these were grouped as a single ROI (CA23DG). ROIs were then projected back into the original three-dimensional dataset for use in averaging. The fMRI time series were divided into $17.5 \mathrm{~s}$ blocks corresponding to each trial. Each subject's responses were classified as either "hit" or "false alarm" for $\mathrm{R}$ and $\mathrm{K}$ trials and as either "correct rejection" or "miss" for not recognized trials. There were too few $\mathrm{R}$ and $\mathrm{K}$ false alarms to allow additional analysis of those trials. Data from the corresponding $17.5 \mathrm{~s}$ of fMRI data for trials of each response type were averaged separately from all voxels in each ROI.

Response amplitudes for both encoding and retrieval were computed for the average event-related responses (unfiltered) by fitting a gamma function to the data (Boynton et al., 1996) using linear regression. Because the timing and shape of the peak response varied somewhat between regions, the gamma fitting procedure included free parameters controlling the delay and shape of the hemodynamic response. These parameters were set separately, for each region, to values that provided the best simultaneous fit to the average data (across subjects) in all conditions. Response amplitudes were measured as the height of the fit response.

For encoding data, fit responses reflected activity $5-6 s$ after stimulus offset, which was so late in the trial that it was not of interest (supplemental Fig. 1, available at www.jneurosci.org as supplemental material). To remove this common late activity, we first subtracted the average time course for forgotten (F) items from each subject's average time course for $\mathrm{R}$ and $\mathrm{K}$ items and then calculated response amplitudes as the maximum of the difference time courses. To discount potential differences in baseline activity between response types, we used the first time point of the difference time course as baseline, which forces the difference time course to begin at zero. This procedure effectively uses the F responses as a model of the late activity to remove it and allow fitting to the early component of the response. We adopted this approach because we did not have a priori hypotheses about the shape of either the late activity or the early responses.

Response amplitudes were entered into a two-way repeated-measures ANOVA, implementing a random-effects model. ANOVAs were conducted separately for encoding and retrieval sessions, and $p$ values were computed using a Greenhouse-Geisser sphericity correction. Simple contrasts between response amplitudes were conducted using paired $t$ tests. The $\alpha$ level was set to 0.05 , and all comparisons were two tailed unless otherwise specified.

\section{Results}

Subjects successfully encoded the stimuli, as reflected by their ability to accurately recognize the cue words during retrieval scans (overall hit rate, 0.62 ; false alarm rate, 0.28 ). For $\mathrm{R}$ responses, the hit rate was $0.29 \pm 0.05$ (mean \pm SEM), and the false alarm rate was $0.02 \pm 0.001$. For $\mathrm{K}$ responses, the hit rate was $0.33 \pm 0.04$, and the false alarm rate was $0.26 \pm 0.04$. Under the assumption that recollection and familiarity are independent processes (Yonelinas, 1994), the mean estimate of the proportion of items accurately recollected was $0.27 \pm 0.05$, and the mean proportion of items accurately judged familiar was $0.17 \pm 0.03$. In the posttest, subjects recalled reliably more details about the study episode for items they had labeled as R than items they had labeled as K (Fig. 1). For both R and K items, subjects were able to indicate the relative position and color of the pictures present during the study phase at above chance levels (for all four tests, $\left.t_{(9)}>2.35 ; p<0.05\right)$. Only for items that had received an $\mathrm{R}$ response were subjects able to recall the word that had been paired with the item during study $\left(t_{(9)}=2.34 ; p<0.05\right)$. In addition, subjects recollected reliably more information about the encoding episode with the $\mathrm{R}$ than the $\mathrm{K}$ response (color, $t_{(9)}=$ $2.66, p<0.05$; orientation, $t_{(9)}=2.63, p<0.05$; word, $t_{(9)}=2.44$, 


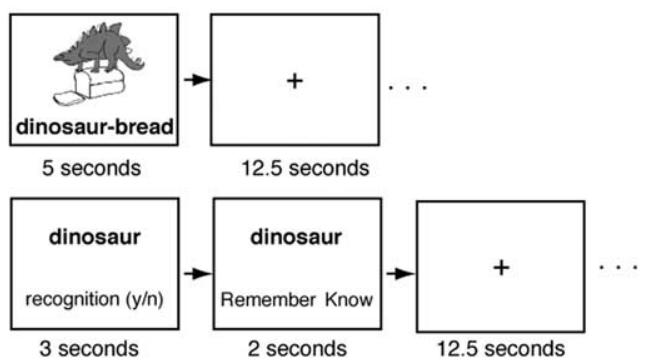

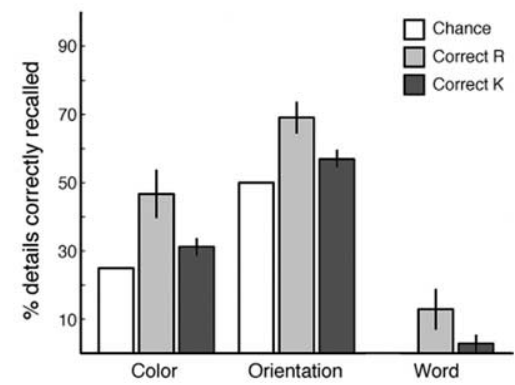

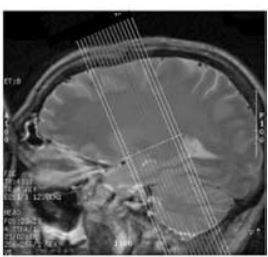

Figure 1. $\quad a$, Sample of the encoding (top) and retrieval (bottom) stimuli and the timing of events within each trial. The color and orientation of the picture pairs varied on each trial and were used to assess recollection in the posttest phase. $\boldsymbol{b}$, Results from the posttest. Chance refers to the probability of guessing correctly for color (4 possible choices) or orientation ( 2 possible choices). $\boldsymbol{c}$, $\mathrm{A}$ sample slice prescription. The slices illustrate the in-plane images that were selected for functional imaging.

$p<0.05)$. Furthermore, subjects were able to retrieve two or more details for a significant number of items that had received an $\mathrm{R}$ response $\left(t_{(9)}=3.16 ; p<0.01\right)$. In contrast, the number of $\mathrm{K}$ items for which subjects could retrieve two or more details on the posttest did not exceed the number expected by chance $\left(t_{(9)}=1.74 ; p>0.05\right)$. Although the posttest does not directly assess the details available to the subject when they made the $\mathrm{R}$ or $\mathrm{K}$ responses previously during scanning, its results suggest that $\mathrm{R}$ responses are associated with the recollection of multiple features bound into a contextual representation. In addition, subjects were faster at making the two decisions leading to an $\mathrm{R}$ response $(2.12 \mathrm{~s}$ ) than they were for the two decisions leading to a $\mathrm{K}$ response $\left(2.48 \mathrm{~s} ; t_{(9)}=4.52 ; p<\right.$ 0.01 ), a correct rejection (CR) of a new item $\left(2.39 \mathrm{~s} ; t_{(9)}=2.74 ; p<0.05\right)$, or the incorrect rejection of an old item $(2.52 \mathrm{~s}$; $\left.t_{(9)}=4.57 ; p<0.01\right)$.

\section{Retrieval}

Our first set of analyses examined patterns of activity across regions during retrieval. Subregions of the hippocampus differed reliably in their responses (two-way ANOVA; subregion vs response type, $F_{(6,48)}=3.83$ and 4.21 for left and right hemispheres; both $p<0.02$ ) (Fig. 2). To further explore these effects within regions, we conducted a series of simple contrasts for each hippocampal region. In the left subiculum, the average MR response amplitude associated with correct $\mathrm{R}$ items was reliably greater than the amplitude associated with correct $\mathrm{K}\left(t_{(8)}=2.39 ; p<\right.$ $0.05)$, forgotten $\left(t_{(8)}=2.70 ; p<0.03\right)$, and correctly rejected items $\left(t_{(8)}=3.92 ; p<0.01\right)$, which did not reliably differ from one another. The right subiculum showed a similar pattern that did not reach statistical significance for all comparisons $(\mathrm{R}>\mathrm{K}$, $\left.t_{(8)}=2.13, p<0.08 ; \mathrm{R}>\mathrm{F}, t_{(8)}=2.40, p<0.05 ; \mathrm{R}>\mathrm{CR}, \mathrm{NS}\right)$. The right CA1 region also showed activity that was mildly consistent with a role in retrieval; $\mathrm{R}$ items produced larger response amplitudes than forgotten items $\left(t_{(8)}=2.36 ; p<0.05\right)$. This pattern of results suggests that regions later in the hippocampal circuit, primarily the sub-
Left CA23DG

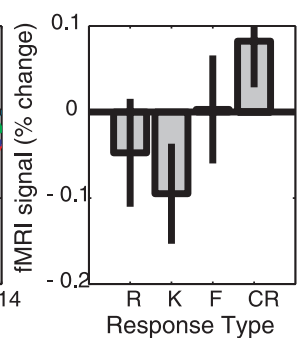

Left CA1
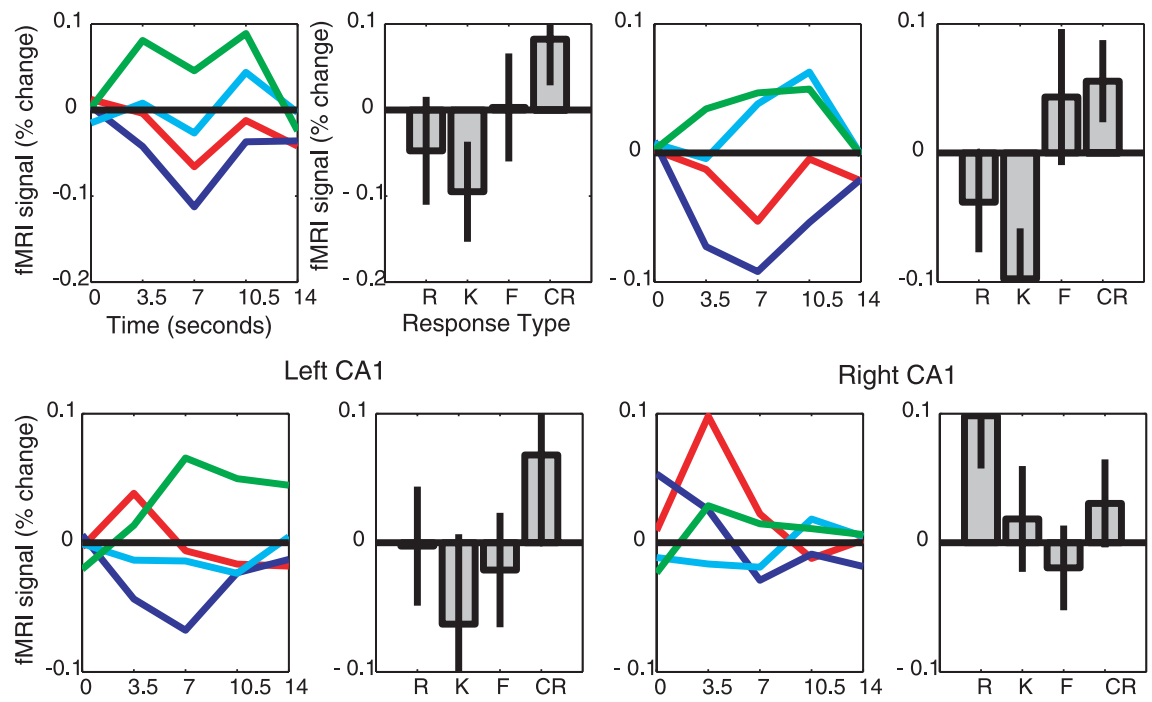

Right CA1
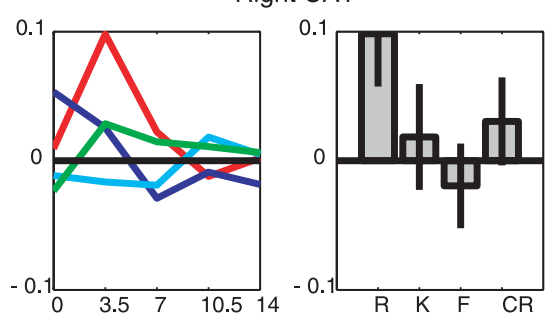

Right subiculum
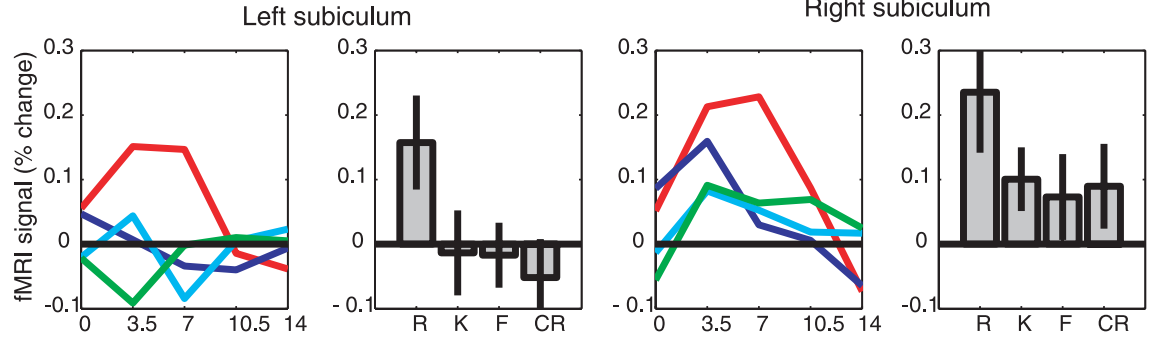
to $\mathrm{R}-\mathrm{K}$ performance during the retrieval phase. The right panels show estimated response amplitudes for each memory type measured by fitting model responses to the data. Amplitude and time course units are both percentage change of the fMRI signal from baseline, and error bars represent \pm 1 SE between subjects. $R$, Red lines; $K$, blue lines; $F$, cyan lines; $C R$, green lines.

iculum and to a lesser extent CA1, are differentially active during the successful recollection of episodes. Our findings extend previous reports of hippocampal involvement in episodic retrieval (Eldridge et al., 2000; Dobbins et al., 2003).

In contrast to the pattern found in the subiculum and CA1, the CA23DG exhibited greater peak activity associated with correctly rejected items than correct $\mathrm{R}$ (right, $t_{(8)}=3.51, p<0.01$; left, NS) or correct K items (right, $t_{(8)}=5.45, p<0.01$; left, $t_{(8)}=$ $3.58, p<0.01)$. This region also showed trends for greater activity for forgotten items than for $\mathrm{R}$ items (right, $t_{(8)}=2.1, p<0.08$; left, NS) and K items (right, $t_{(8)}=3.45, p<0.01$; left, $t_{(8)}=2.18$, 
Left Perirhinal
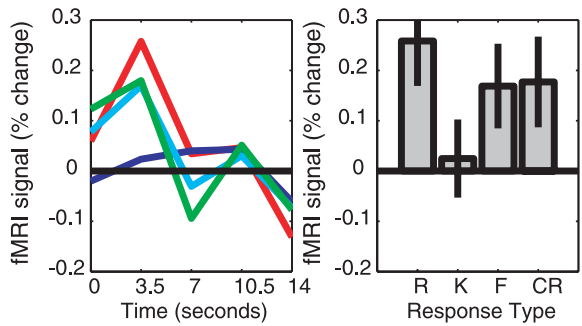

Left PHC
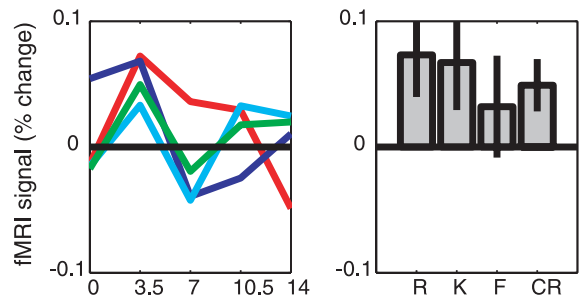

Left fusiform
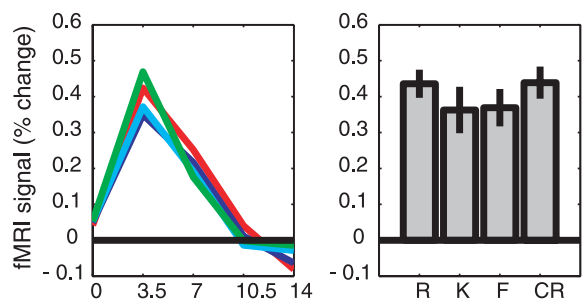

Right Perirhinal
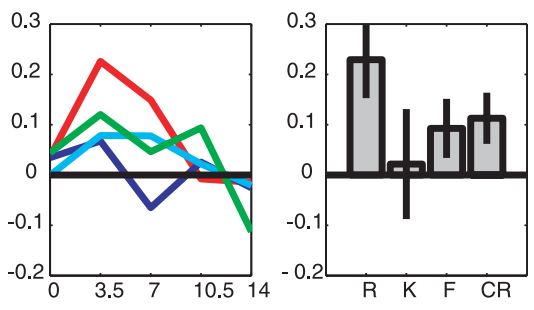

Right PHC
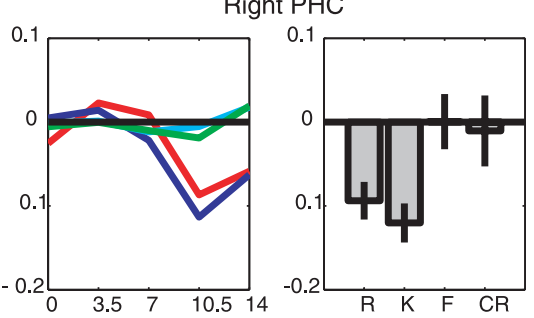

Right fusiform
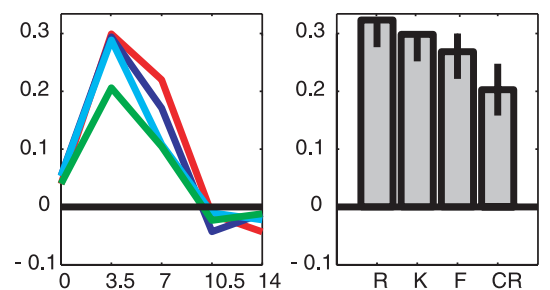

Figure 3. Averaged event-related responses in anatomically defined extrahippocampal regions from nine subjects sorted according to R-K performance during the retrieval phase. Plotting conventions are as in Figure 2. PHC, Parahippocampal cortex.

$p<0.08)$. Thus, CA23DG region activity was greater for new items and items judged new than for recognized items (for a further discussion of this finding, see below).

To examine differences across regions for recollection and familiarity-based recognition, we conducted ANOVAs separately for $\mathrm{R}$ and $\mathrm{K}$ trials. For $\mathrm{R}$ response trials, there was a significant effect of subregion on activation level in the right hemisphere $\left(F_{(2,16)}=\right.$ 9.31; $p<0.01$ ). Post hoc comparisons revealed that this effect was attributable to greater activation in the subiculum than in the other two subregions $\left(F_{(1,8)}=8.89 ; p<0.02\right)$. In the left hemisphere, there was a similar trend that did not reach statistical significance $\left(F_{(2.16)}=\right.$ $3.11 ; p<0.08)$.

For $\mathrm{K}$ response trials, there was a significant effect of subregion on level of activation in the right hemisphere only $\left(F_{(2,16)}=\right.$ $7.86 ; p<0.01)$. Post hoc comparisons showed that this effect was attributable to the deactivation from baseline in the CA23DG compared with the other two regions $\left(F_{(1,8)}=13.88 ; p<0.01\right)$. For K response trials, no hippocampal subregion showed statistically reliable activation above baseline.

MTL regions outside the hippocampus were also active during retrieval (Fig. 3). For each of these regions, we performed contrasts comparing activation levels for the different response types. The left perirhinal cortex showed greater response amplitudes for $\mathrm{R}$ items than for $\mathrm{K}$ items $\left(t_{(8)}=2.86 ; p<0.02\right)$. Responses for correctly rejected and forgotten items were intermediate in size and did not differ reliably from either $\mathrm{R}$ or $\mathrm{K}$ responses. In the right fusiform gyrus, correctly rejected items produced less activity than the other types of items $\left(t_{(8)}=4.22,2.95,2.05 ; p<0.01\right.$, $0.025,0.08$ for $\mathrm{R}, \mathrm{K}$, and forgotten items, respectively). Finally, in the right parahippocampal cortex, both $\mathrm{R}$ and $\mathrm{K}$ items produced large negative responses late in the trial that were larger than responses for forgotten items $\left(t_{(8)}=3.34\right.$ and $\left.3.33 ; p<0.02\right)$.

The results from the retrieval scans indicated that activation was greater in the CA23DG region for unrecognized items than for recognized items. Previous neuroimaging studies of the hippocampus have revealed increased activation for novel items compared with repeated items, suggesting that the hippocampus is important for registering unexpected events (Strange and Dolan, 2001). Alternatively, hippocampal activity associated with novel items may reflect encoding processes. Novel items would be predicted to require more extensive encoding than previously seen items (Tulving et al., 1996). Stark and Okado (2003) showed that hippocampal activation associated with novel items on a recognition test predicted later memory for those items, supporting the idea that this activation is related to successful encoding. In the present study, the increased activation seen in the CA23DG region for novel items is consistent with the occurrence of encoding these items during the retrieval phase (Fig. 2). The pattern of activity suggests that the presentation of either a truly new item or an item perceived to be new may recruit the CA23DG to create a memory representation. Our results specify more precisely the location of hippocampal regions that have been shown previously to be engaged during encoding of novel items (Stern et al., 1996).

\section{Encoding}

To examine whether the CA23DG region is involved in the creation of new memories, we analyzed fMRI responses from the encoding scans, averaging them based on subsequent memory performance. An initial analysis did not find evidence for differences in response between regions, but closer examination of the fMRI time courses showed that differences between remembered and forgotten items early in the trial were being masked by activity that appeared late in the trial (supplemental Fig. 1, available at www.jneurosci.org as supplemental material). To reveal these early differences, we subtracted subjects' average time courses for items that would later be forgotten from their time courses for items that would later be recognized (separately for items that received $\mathrm{R}$ and $\mathrm{K}$ responses) (Fig. 4). An analysis of the effects of response type ( $\mathrm{R}$ vs $\mathrm{K}$ ) and hippocampal subregion on these subtracted time courses revealed, for the left hemisphere, a significant interaction $\left(F_{(2,16)}=7.05 ; p<0.01\right)$.

We next directly examined whether activation in the CA23DG region was associated with memory encoding, as suggested by the pattern of results found during retrieval. We used simple contrasts to determine whether activation in the CA23DG during encoding was greater for items subsequently labeled as $\mathrm{R}$ or $\mathrm{K}$ compared with items that were subsequently forgotten. These planned comparisons were one tailed because they test an a priori hypothesis derived from the retrieval results. They revealed that, in the left CA23DG, items that were later labeled as either R or K 
led to greater early responses than items that would be forgotten $\left(\mathrm{R}, t_{(8)}=1.98, p<\right.$ $\left.0.05 ; \mathrm{K}, t_{(8)}=3.17, p<0.05\right)$. This pattern of activity suggests that the CA23DG region contributes to successful memory encoding.

We then performed contrasts for the other hippocampal subregions to identify additional regions potentially associated with successful encoding. In the CA1 region, the activation during encoding for items that were later forgotten or successfully recognized was small and not statistically reliable. In the subiculum, there was a different pattern of results than in the CA23DG region. In the left subiculum, the response amplitudes for recognized and forgotten items were only different for items recognized based on familiarity $\left(t_{(8)}=3.92 ; p<0.05\right)$. Response amplitudes in the subiculum were also greater for items recognized based on familiarity than for items that were recollected $\left(t_{(8)}=\right.$ $2.49 ; p<0.05)$ Although these results suggest that the left subiculum may play a role in the encoding of information that later supports familiarity judgments, no evidence was found that this region encodes information that supports recollection.

Encoding-related responses were evident bilaterally in a subset of the extrahippocampal MTL regions (Fig. 5). Unlike in the hippocampus, the late activation peak was not present. Thus, we were able to directly compare peak activation during encoding for subsequently remembered and forgotten responses. These analyses revealed that the right fusiform gyrus and the right parahippocampal cortex showed activation that was tied to subsequent memory. Items that would later be given an $\mathrm{R}$ response led to greater activation at encoding than items that would later be forgotten (parahippocampal cortex, $t_{(8)}=3.01, p<0.02$; fusiform, $t_{(8)}=3.13 p<0.02$ ). This pattern is consistent with previous studies examining encoding-related activation for both words (Wagner et al., 1998) and pictures (Brewer et al., 1998).

To compare directly the pattern of activation during encoding and retrieval of episodic memories, we performed a subregion $\times$ task phase (encoding vs retrieval) ANOVA on response amplitudes for R trials. This analysis compared activation related to successful recollective encoding to activation associated with successful recollection across hippocampal subregions and yielded an interaction between these two factors in the left hemisphere $\left(F_{(2,16)}=4.87 ; p<\right.$ $0.05)$. This interaction confirms the differential activity of the CA23DG and subicular regions during these two phases of episodic memory processing.

\section{Discussion}

The present data reveal a dissociation between episodic encoding and retrieval processes within the hippocampus. A subregion early in the hippocampal circuit (CA23DG) was engaged by processes related to the creation of a memory representation, although this region was not selectively involved in the retrieval of old memories. In contrast, a subregion later in the hippocampal circuit (the subiculum) was engaged during episodic retrieval but
Left CA23DG

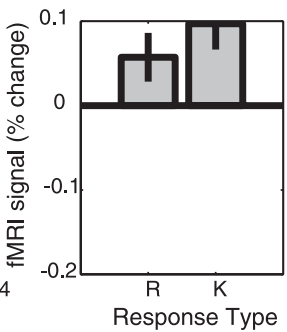

Left CA1

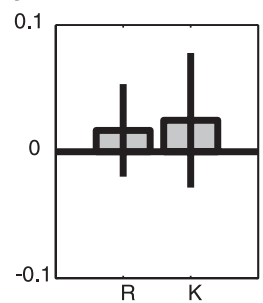

Left subiculum

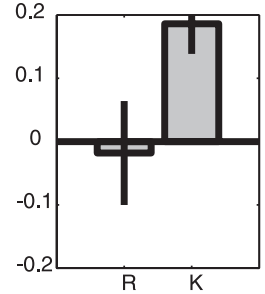

Right CA23DG
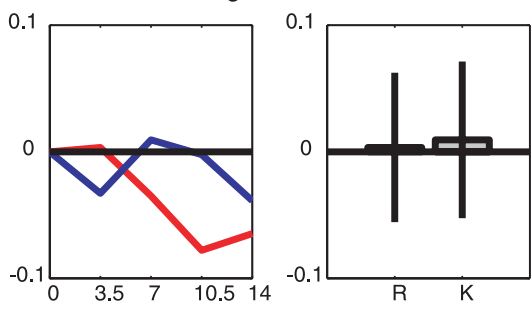

Right CA1
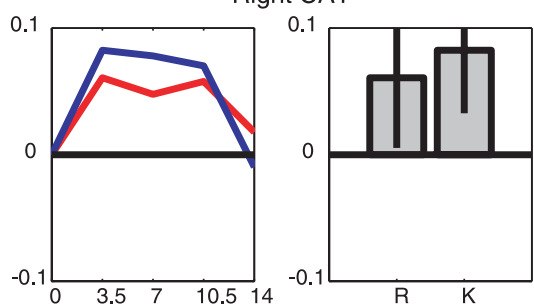

Right subiculum
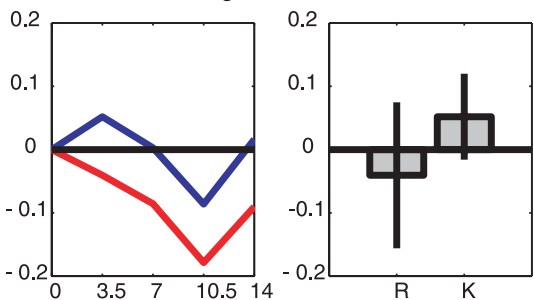

Figure 4. Averaged encoding event-related responses from nine subjects sorted according to whether items would later be responses from the $R$ and $K$ responses. This subtraction was performed to remove the late activity and reveal potential differences between conditions in the early part of the trial. $R$, Red lines; $K$, blue lines. The right panels plot estimated response amplitudes from the difference time courses.

not during the successful encoding of episodic memories. The dissociation we observe between encoding and retrieval does not simply reflect differences in the materials presented during the two scanning sessions. For example, the CA23DG not only showed greater activity during picture-word encoding trials that led to better memory but also showed greater activity during retrieval trials when novel or forgotten words were presented. Thus, the CA23DG appears to be important for encoding regardless of whether words are presented alone (our retrieval scans) or paired with pictures (our encoding scans).

Previous neuroimaging work has found activity in the hippocampus during both encoding and retrieval. Two previous studies using block designs found activity related to retrieval in the subiculum (Gabrieli et al., 1997; Zeineh et al., 2003). Our results indicate that retrieval-related activity in the subiculum may be specific for episodic memories. We also find activity related to encoding in the CA23DG region, consistent with the recent finding that activity in this region correlates with incremental learning for face-name pairs (Zeineh et al., 2003). Our data extend these previous results by finding a trial-by-trial relationship between successful encoding and early activity in the CA23DG. Our results also agree with a number of previous studies reporting anatomically unspecified hippocampal activity during encoding (Otten et al., 2001; Davachi and Wagner, 2002; Strange et al., 2002).

In the present study, CA23DG activation during encoding was associated with both recollection and familiarity-based recognition on the subsequent memory test. There is evidence that the 

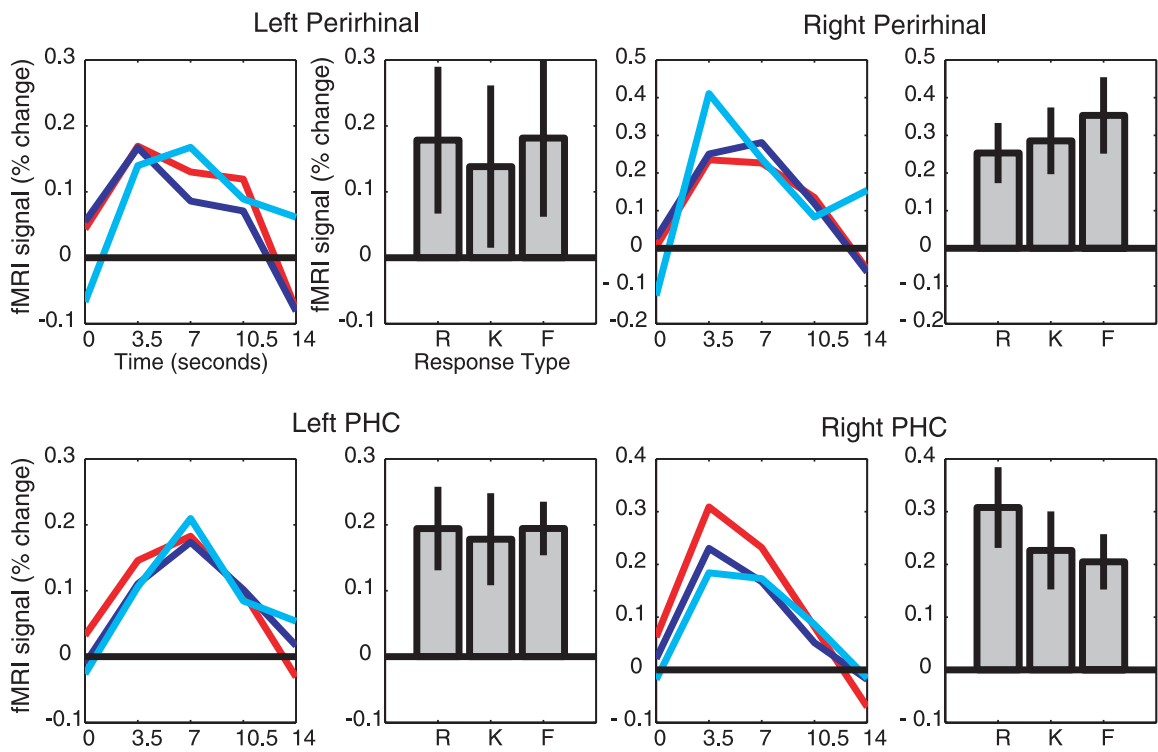

Left PHC
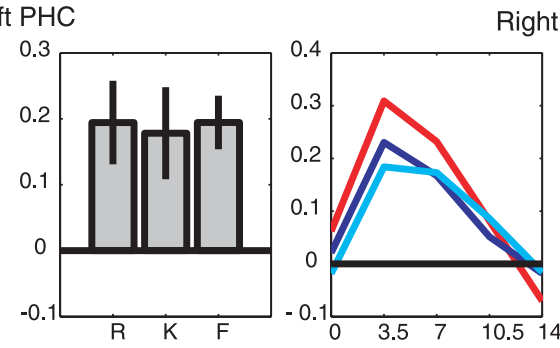

Right PHC
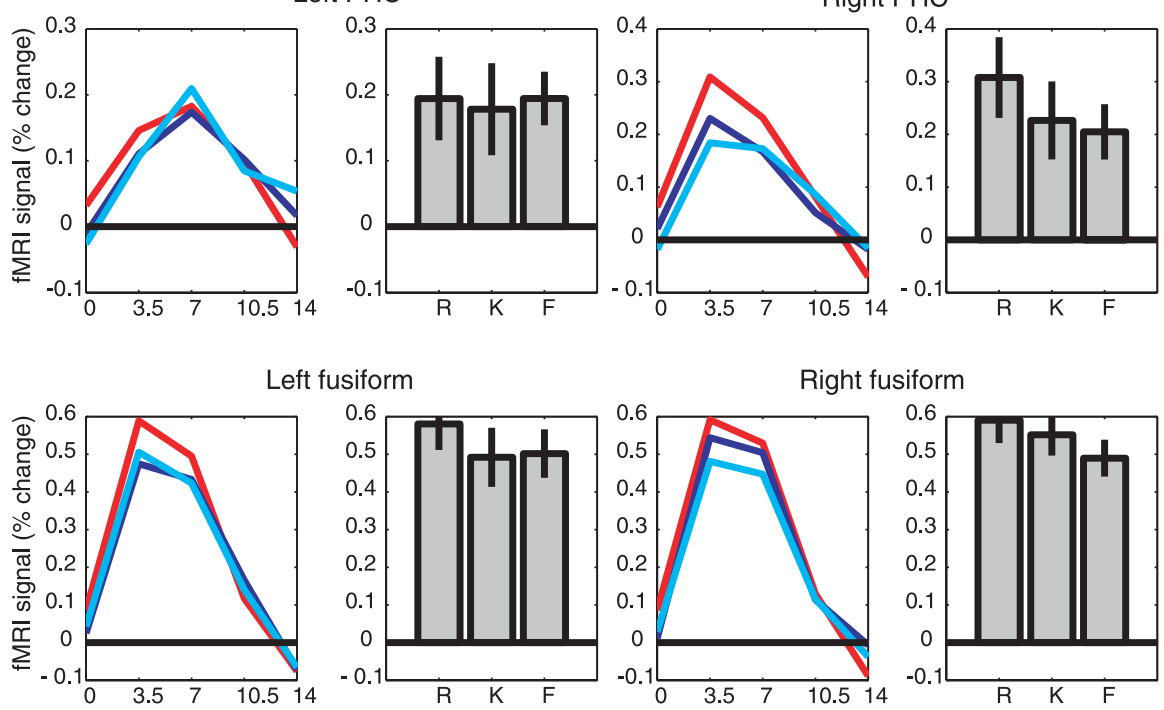

Figure 5. Averaged event-related responses in anatomically defined extrahippocampal regions from nine subjects sorted according to R-K performance during the encoding phase. Because late peaks do not dominate as in the hippocampus, simple average time courses are plotted and were used to estimate response amplitudes (not difference time courses as in Fig. 4). Plotting conventions are as in Figure 2.

hippocampus may be particularly involved in encoding episodic memories (Ranganath et al., 2004). It is possible that, with the relatively long study-test interval used in the present study $(24 \mathrm{~h})$, at least some of the items that had been initially encoded as episodic memories were judged merely familiar at the time of test. Thus, the present results are not inconsistent with the idea that the hippocampus has a specific role in the encoding of episodic memories. The present results also provide some support for the idea that encoding for later familiarity can rely on neural circuitry that is distinct from that involved in the encoding of episodic memories. Although the subiculum does not appear to be associated with the formation of episodic memories, it does appear to be active during the encoding of items that will be judged familiar. It is possible that the subiculum and associated cortical regions are part of the neural substrate of familiarity encoding. It is also possible that this activity in the subiculum reflects episodic retrieval during the encoding phase. It may be that, in this paradigm, such retrieval resulted in more familiarity for these items at test without better memory for individuating information from the study phase associated with these items.

Hippocampal activation has been associated with both the encoding of novel items and the recollection of old items. Indeed, the increase in BOLD signal to both recognized items and novel items during a recognition test may have made the observation of retrieval-related activity in the hippocampus more difficult (Stark and Okado, 2003). By identifying the differential contributions of subcomponents within the hippocampal region, we have been able to disentangle the neural substrates of encoding and retrieval. These data may constrain developing theories of the neural computations performed by each subregion (Hasselmo et al., 2002; Norman and O'Reilly, 2003). For example, the increase in activation in CA23DG during successful encoding suggests that the formation of memory representations is accompanied by specific increases in synaptic activity in this region.

\section{References}

Amaral DG (1993) Emerging principles of intrinsic hippocampal organization. Curr Opin Neurobiol 3:225-229.

Boynton GM, Engel SA, Glover GH, Heeger DJ (1996) Linear systems analysis of functional magnetic resonance imaging in human V1. J Neurosci 16:4207-4221.

Brewer JB, Zhao Z, Desmond JE, Glover GH, Gabrieli JD (1998) Making memories: brain activity that predicts how well visual experience will be remembered. Science 281:1185-1187.

Davachi L, Wagner AD (2002) Hippocampal contributions to episodic encoding: insights from relational and item-based learning. J Neurophysiol 88:982-990.

Dobbins IG, Rice HJ, Wagner AD, Schacter DL (2003) Memory and orientation success: separable neurocognitive components underlying episodic recognition. Neuropsychologia 41:318-333.

Eldridge LL, Knowlton BJ, Furmanski CS, Bookheimer SY, Engel SA (2000) Remembering episodes: a selective role for the hippocampus during retrieval. Nat Neurosci 3:1149-1152.

Eldridge LL, Sarfatti S, Knowlton BJ (2002) The effect of testing procedure on remember-know judgments. Psychon Bull Rev 9:139-145.

Gabrieli JD, Brewer JB, Desmond JE, Glover GH (1997) Separate neural bases of two fundamental memory processes in the human medial temporal lobe. Science 276:264-266.

Gadian DG, Aicardi J, Watkins KE, Porter DA, Mishkin M. Vargha-Khadem F (2000) Developmental amnesia associated with early hypoxicischaemic injury. Brain 123:499-507.

Greicius MD, Krasnow B, Boyett-Anderson JM, Eliez S, Schatzberg AF, Reiss AL, Menon V (2003) Regional analysis of hippocampal activation during memory encoding and retrieval: fMRI study. Hippocampus 13:164-174.

Hasselmo ME, Bodelon C, Wyble BP (2002) A proposed function for hippocampal theta: separate phases of encoding and retrieval enhance reversal of prior learning. Neural Comput 14:793-818.

Henson RN, Rugg MD, Shallice T, Josephs O, Dolan RJ (1999) Recollection and familiarity in recognition memory: an event-related functional magnetic resonance imaging study. J Neurosci 19:3962-3972.

Kopelman MD, Stanhope N, Kingsley D (1999) Retrograde amnesia in patients with diencephalic, temporal lobe or frontal lesions. Neuropsychologia 37:939-958.

Lepage M, Habib R, Tulving E (1998) Hippocampal PET activations of memory encoding and retrieval: the HIPER model. Hippocampus 8:313-322.

Norman KA, O’Reilly RC (2003) Modeling hippocampal and neocortical contributions to recognition memory: a complementary learning systems approach. Psychol Rev 110:611-646.

Otten LJ, Henson RN, Rugg MD (2001) Depth of processing effects on neural correlates of memory encoding: relationship between findings from across- and within-task comparisons. Brain 124:399-412.

Ranganath C, Yonelinas AP, Cohen MX, Dy CJ, Tom SM, D’Esposito M 
(2004) Dissociable correlates of recollection and familiarity within the medial temporal lobes. Neuropsychologia 42:2-13.

Reed JM, Squire LR (1998) Retrograde amnesia for facts and events: findings from four new cases. J Neurosci 18:3943-3954.

Rempel-Clower NL, Zola SM, Squire LR, Amaral DG (1996) Three cases of enduring memory impairment after bilateral damage limited to the hippocampal formation. J Neurosci 16:5233-5255.

Schacter DL, Wagner AD (1999) Medial temporal lobe activations in fMRI and PET studies of episodic encoding and retrieval. Hippocampus 9:7-24.

Schacter DL, Curran T, Reiman EM, Chen K, Bandy DJ, Frost JT (1999) Medial temporal lobe activation during episodic encoding and retrieval: a PET study. Hippocampus 9:575-581.

Snodgrass JG, Vanderwart M (1980) A standardized set of 260 pictures: norms for name agreement, image agreement, familiarity, and visual complexity. J Exp Psychol [Human Learn] 6:174-215.

Stark CE, Okado Y (2003) Making memories without trying: medial temporal lobe activity associated with incidental memory formation during recognition. J Neurosci 23:6748-6753.

Stern CE, Corkin S, Gonzalez RG, Guimaraes AR, Baker JR, Jennings PJ, Carr CA, Sugiura RM, Vedantham V, Rosen BR (1996) The hippocampal formation participates in novel picture encoding: evidence from functional magnetic resonance imaging. Proc Natl Acad Sci USA 93:8660-8665.

Strange BA, Dolan RJ (2001) Adaptive anterior hippocampal responses to oddball stimuli. Hippocampus 11:690-698.

Strange BA, Otten LJ, Josephs O, Rugg MD, Dolan RJ (2002) Dissociable human perirhinal, hippocampal, and parahippocampal roles during verbal encoding. J Neurosci 22:523-528.

Tulving E, Markowitsch HJ, Craik FE, Habib R, Houle S (1996) Novelty and familiarity activations in PET studies of memory encoding and retrieval. Cereb Cortex 6:71-79.

Vargha-Khadem F, Gadian DG, Watkins KE (1997) Differential effects of early hippocampal pathology on episodic and semantic memory. Science 277:376-380.

Wagner AD, Schacter DL, Rotte M, Koutstaal W, Maril A, Dale AM, Rosen BR, Buckner RL (1998) Building memories: remembering and forgetting of verbal experiences as predicted by brain activity. Science 281:1188-1191.

Woods RP, Dapretto M, Sicotte NL, Toga AW, Mazziotta JC (1999) Creation and use of a Talairach-compatible atlas for accurate, automated, nonlinear intersubject registration, and analysis of functional imaging data. Human Brain Mapp 8:73-79.

Yonelinas AP (1994) Receiver-operating characteristics in recognition memory: evidence for a dual process model. J Exp Psychol Learn Mem Cogn 20:1341-1354.

Zeineh MM, Engel SA, Bookheimer SY (2000) Application of cortical unfolding techniques to functional MRI of the human hippocampal region. NeuroImage 11:668-683.

Zeineh MM, Engel SA, Thompson PM, Bookheimer SY (2001) Unfolding the human hippocampus with high resolution structural and functional MRI. Anat Rec 265:111-120.

Zeineh MM, Engel SA, Thompson PM, Bookheimer SY (2003) Dynamics of the hippocampus during encoding and retrieval of face-name pairs. Science 299:577-580.

Zola-Morgan S, Squire LR, Amaral DG (1986) Human amnesia and the medial temporal region: enduring memory impairment following a bilateral lesion limited to field CA1 of the hippocampus. J Neurosci 6:29502967. 